Proceedings of the 2011 Winter Simulation Conference

S. Jain, R.R. Creasey, J. Himmelspach, K.P. White, and M. Fu, eds.

\title{
EXTENDSIM TECHNOLOGY: SCENARIO MANAGEMENT
}

\author{
David Krahl \\ Imagine That, Inc \\ 6830 Via Del Oro, Suite 230 \\ San Jose, CA 95030, USA
}

\begin{abstract}
Simulation models are typically built to obtain an understanding of the system dynamics and compare alternatives. ExtendSim's Scenario Manager provides an easy interface to evaluate different model configurations and explore the effects of model parameters. Scenario management provides for a systematic, controlled approach to the investigation of a system or process. Among other things, it can be used for problem solving ("what are the main factors contributing to the problem"), parameter design ("how well does the system/process perform given specified factors"), and robustness studies ("what is the best configuration of factor values to maximize/minimize variations in response"). Thus it has a very broad application across all disciplines.
\end{abstract}

\section{INTRODUCTION}

ExtendSim is an advanced simulation environment that supports discrete event, continuous, discrete rate, and agent based technologies. Models are comprised of blocks that communicate with each other to describe the simulated sequence and the general logic of the model. General purpose blocks are provided with ExtendSim. For specialized purposes, these blocks can be modified or new ones created by the end user. New blocks can be either programmed in ExtendSim's C-based ModL language or by combining existing blocks together. Regardless of the creation method, blocks can be stored in libraries for use in other models. Additional tools are provided to manage the data so that blocks with the same structure can reference different data sets.

ExtendSim pioneered the message based architecture (Krahl and Lamperti 1997) found in modern simulation tools. When an event or state change occurs, messages are broadcast to any impacted blocks. There are three mechanisms for message transmission: through connections; directly from block to block; and even through ExtendSim's graphical simulation database (Diamond et al. 2010).

ExtendSim has also lead other simulation software in data management techniques and mechanisms. ExtendSim's integrated simulation database facilitates a more organized and scalable modeling approach. A variety of simulation-specific features have been included in ExtendSim's database that make it uniquely useful for simulation modeling.

ExtendSim's Scenario Manager leverages the database to store model factors and responses. This structure maintains an organizational scheme and allows straightforward access to the model data. The database also facilitates communication between ExtendSim and other analysis programs. Because of this it is easy to use an outside statistical package or spreadsheet to analyze the relationship between the model factors and responses. 


\section{EXTENDSIM STRUCTURE}

An ExtendSim model is created by adding blocks to a model worksheet, connecting them together, and entering the simulation data. Each type of block has its own functionality, dialog, help, icon, and connections. Each instance of a block in the model has its own data. Blocks perform a number of functions in a simulation model including:

- Simulating the steps in a process (Queue, Activity)

- Performing a calculation (Math, Random Number, Equation)

- Interfacing with other applications or data storage (Read, Write)

- Providing a model utility (Find and Replace, Count Blocks)

- Plotting model results (Plotter, Histogram)

- Animating (Animate Value, 3D Scenery)

- Creating a user interface (Popup, Buttons)

The logical entity that moves through the system is referred to as an item. Items carry properties or attributes with them as they progress from one block to the next. Items are represented using lightweight data structures allowing virtually unlimited numbers to exist simultaneously within a model.

The source code for all of these blocks is available and can be viewed or modified by the end user. Blocks can be created from existing blocks or created from scratch. This allows the modeler or developer, to create their own libraries for specific modeling domains. A whole range of simulation products has been created based on the ExtendSim engine. These include applications for pulp and paper manufacturing, chemical process control, biological processes, and analytical chemistry.

Hierarchical blocks contain other blocks (either programmed or hierarchical). This helps to organize the model. Hierarchical blocks can be stored in a library and reused in the same or different models. ExtendSim has tools for creating an interface within the hierarchical block, making it easy for the modeler to expose important parameters and results.

ExtendSim's database is an integrated part of the model structure. Many of the modeling components are designed around the database to store and manage data. The database can also be used as part of the fabric of the simulation. As a database record value changes, interested blocks will receive a message notifying them of the change so that they can react accordingly. This powerful capability simplifies communication in complex models, eliminating cumbersome end-of-event status checks and event polling (Diamond et al. 2010).

\section{SCENARIO MANAGEMENT AND DESIGN OF EXPERIMENTS}

One of the primary purposes of a simulation model is to discover the responses of the simulated system based on different factor values. Improvements in the performance and memory capacity of personal computers have made it possible to automate this process. In addition, external analysis tools such as Minitab ${ }^{\circledR}$ Statistical Software and JMP ${ }^{\circledR}$ can be used to generate designs. Microsoft Excel ${ }^{\circledR}$, Minitab, $\mathrm{JMP}$, and $\mathrm{R}$ as well as other applications can be used to analyze the simulation results.

With the improvements in performance of modern computers, model size has been increasing as well. Simulation software performance and model efficiency continue to be important factors. When analyzing the model responses, it can be useful to utilize design of experiments (DOE) technology to reduce the number of model runs required to compare multiple scenarios. This is particularly useful for initial investigations where the modeler needs to determine which factors have the most impact on system performance. The modeler can then focus on a detailed analysis of the model by varying only the parameters that have a measurable impact on the results. 


\section{THE SCENARIO MANAGER}

There are a variety of methods that the modeler can use to manage model configurations in ExtendSim. For most purposes, ExtendSim's dedicated Scenario Manager block will be the tool of choice. The Scenario Manager is implemented as a block in the Value library. It is available in the ExtendSim AT and ExtendSim Suite packages. This block provides a straightforward interface to dialog values from other blocks and the ExtendSim database as well as a recording mechanism for the model responses and an export tool so that the factors and responses can be analyzed in other software programs.

ExtendSim's Scenario Manger capitalizes on ExtendSim's open architecture. Because it is implemented as a block, the source code is open to the modeler to view or potentially modify. For example, a researcher may want to study a new experimental design algorithm. The researcher has access to the Clanguage based ModL source code of the Scenario Manager and can add additional types of experimental designs.

In addition, the Scenario Manager is designed for interactive execution. The results are displayed as the model runs and the set of results that are displayed can be changed "on-the-fly." During the run the modeler sees the statistics for the responses change as each run completes and can view the individual response values.

For analysis, the Scenario Manager seamlessly communicates with other simulation programs such as Excel, JMP, and Minitab. With a few mouse-clicks you can export the scenario factors and all of the responses to an outside application. You can even configure the model to export the results automatically when the scenario runs have completed. By leveraging advanced analysis programs, ExtendSim users have easy access to powerful statistical tools and presentation programs. In fact, a menu selection and a single mouse click can generate the experimental design, run the scenarios, and export the results to JMP or Minitab.

\subsection{Model Factors}

The Scenario Manager uses a standard design of experiments (DOE) nomenclature. Model inputs are referred to as factors and the simulation results are responses. A unique feature is that factors can be databases, tables, fields, or records as well as single dialog variables. A dialog variable is a single numerical value from any block in the model. It can be added to the Scenario Manager by either dragging a clone (using ExtendSim's clone tool) of the dialog item to the icon of the Scenario Manager or by shift clicking on the dialog item and selecting "add factor".

Database factors and responses are entered by selecting from popup menus in the Scenario Manager's database factors and responses dialogs. Figure 1 shows the dialog where scenario factors have been entered. The information for each dialog factor includes its name, where it comes from (block name, block number, block label, hierarchical block, the dialog variable name, and the row and column), its current value and the range of values that can be assigned (minimum, maximum and step). The range for each factor is used for the automatic generation of DOE scenarios. For database table factors, a source list table is used to provide a list of the possible tables that can be used as factors. At the start of each scenario, one source table for each database factor is copied into a target table which is used by the model for its calculations.

In Figure 2 the relationship between the three types of tables is illustrated. The scenarios are created for each database factor based on the contents of the source list table. While tables have been used in this example, database factors can also be records, fields, or even entire databases. In the case of fields or records, the source list is implied as all of the fields in a table or all of the records in a field respectively. In this case, there are two source tables that can be substituted into the target table for a given scenario. At the start of each scenario run, the contents of one of the source tables is copied into the target table. This design allows the modeler to flexibly create a set of scenarios that not only incorporate scalar model parameters but entire data sets as well. 


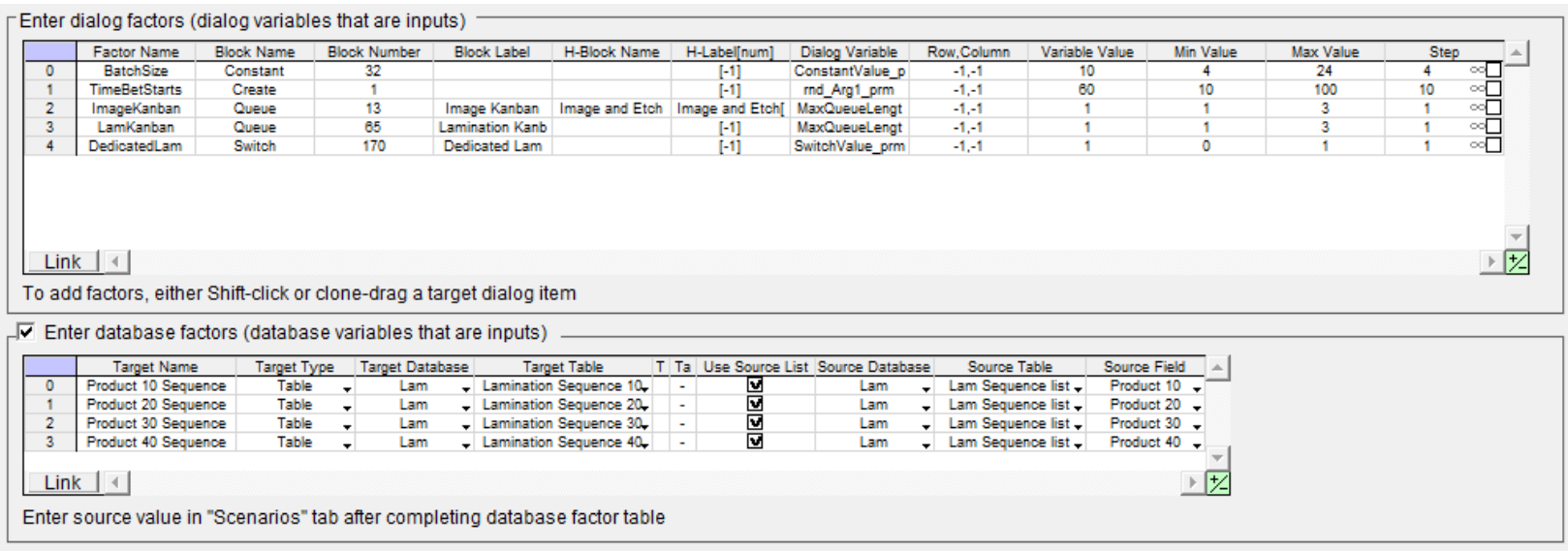

Figure 1: Factor dialog for Scenario Manager

Target tables

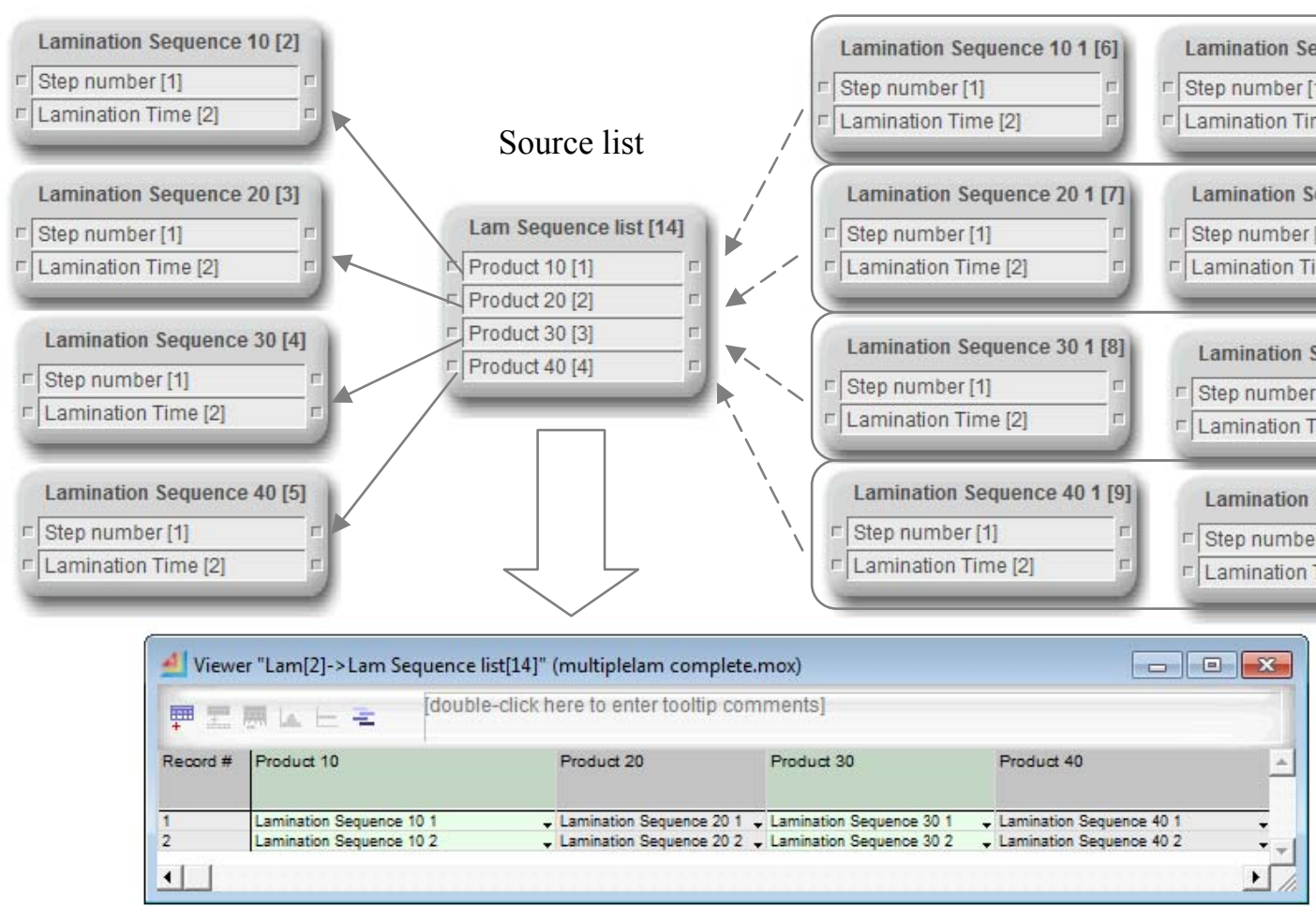

Figure 2: Database factors

\subsection{Model Responses}

Like model factors, any model responses or performance measures can be added to the Scenario Manager by shift-clicking on a dialog item and selecting "Scenario Manager: Add response." This inserts a row into the response table and enters the appropriate block and dialog item information. 


\section{Krahl}

In addition to the block dialog information, the modeler can add additional information for automated DOE generation. These include whether it is desirable to minimize or maximize the response, the statistics that are calculated and whether or not the response will be included in reports.

You can also create database responses that are generated for every run and scenario (Figure 3). These can be used to store detailed results and logs from the simulation.

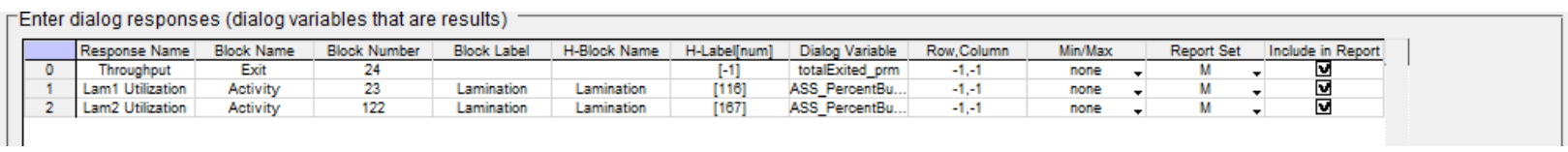

Figure 3: Responses

\subsection{Scenarios}

The Scenarios tab contains the values for the model factors and, after the scenarios have been run, a statistical summary of the responses. The individual results for every response in every scenario run are stored in ExtendSim's graphical simulation database.

The heart of the Scenario Manager is the Scenarios tab (Figure 4). Here, the modeler sets the run parameters, creates the scenario, runs the simulations and monitors the progress of the scenarios as they run. As the scenarios run, a status displays a bar graph of the progress so far as well as the number of runs and scenarios completed.

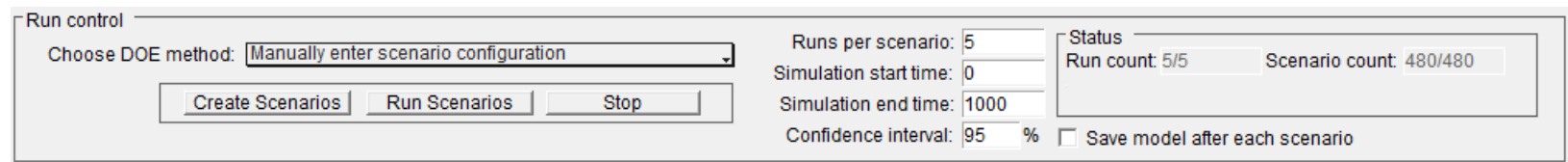

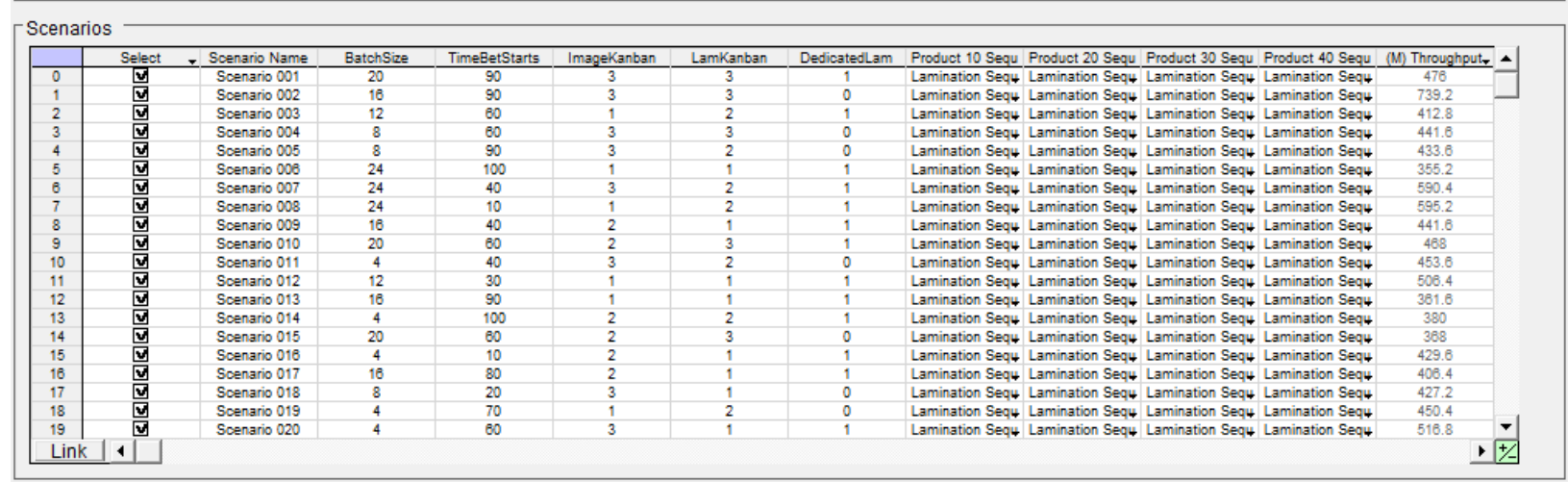

Figure 4: Scenarios tab

Scenarios can be created manually or by using an automatic method including a full factorial design, a JMP custom design, or Minitab optimal design (Figure 5). Designs from other sources can be pasted into the Scenario table. Any number of designs can be evaluated by the Scenario Manager. If one of the "Create and immediately run..." options is used, the experimental design is generated, the scenarios are immediately generated and the responses are automatically exported back to the DOE (JMP or Minitab) software. This is a one-step method for performing scenario analysis. Because the Scenario Manager is designed to be "hooked-up" to any statistical analysis program that supports the COM or .net technology, other interfaces may be developed in the future or can be developed by the modeler. 


\section{Manually enter scenario configuration}

\section{Full factorial design}

JMP custom design

Create and immediately run JMP custom design

Minitab optimal design

Create and immediately run Minitab optimal design

Figure 5: Scenario generation methods

Once the scenarios have been created they can be run and their progress monitored. Figure 6 shows the scenario status dialog found on the Scenarios tab. If necessary, the scenario runs can be stopped, the computer turned off and the execution of the scenarios can be resumed later. The Scenario Manger tracks which scenarios have been completed and will resume where the runs left off.

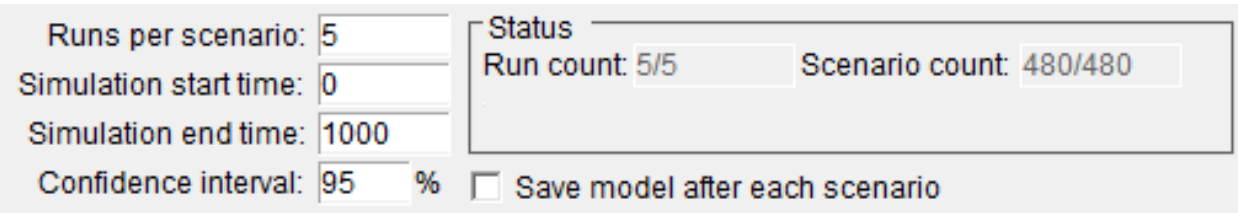

Figure 6: Scenario Manager run control and status

The statistics that are displayed in the Scenarios table can be controlled. Because the individual results are stored in ExtendSim's database, the result sets can be chosen before, after, and even during the scenario runs. The results are displayed immediately and are updated while the model is running. There is no need to wait until the completion of all of the scenario runs to see the intermediate results. Figure 7 contains the list of result sets available. Each of these is selectable on a response-by-response basis.

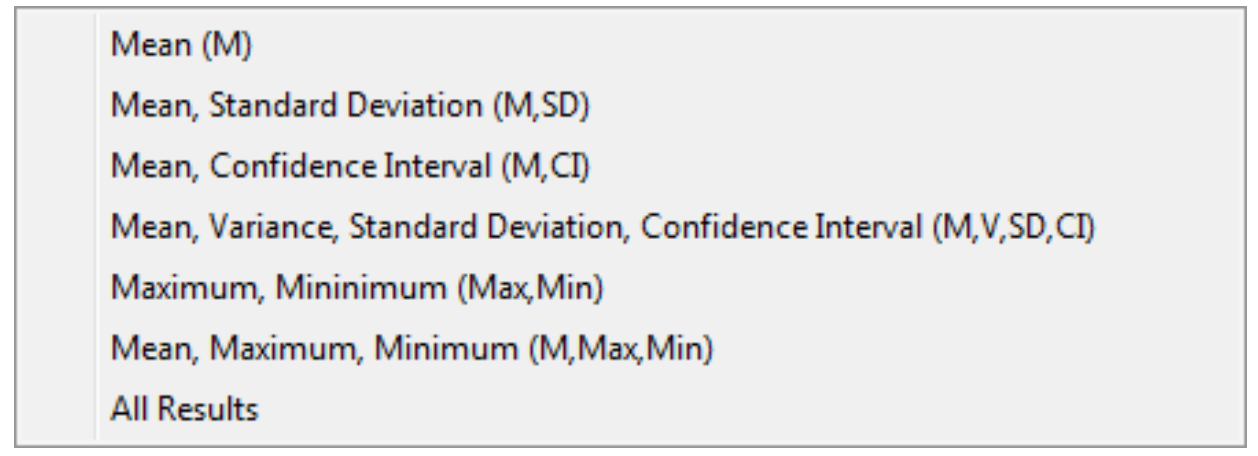

Figure 7: Result sets

In Figure 8 we have selected "All results" for the throughput response. Here the mean (M), variance (V), standard deviation (SD), maximum (Max), minimum (Min) and confidence interval (CI) are displayed based on the results of multiple simulation runs.

By clicking on the "Show" button in the Scenarios table, the individual response values for that scenario are displayed. Figure 9 shows the response values for an individual scenario. A statistical summary of this information is displayed in the Scenarios table. 
Krahl

\begin{tabular}{|c|c|c|c|c|c|}
\hline (M) Throughput. & (V) Throughput. & (SD) Throughput & (Max) Throughput & (Min) Throughput & (CI) Throughput \\
\hline 476 & 7480 & 86.486993241759 & 600 & 380 & 107.38778581546 \\
\hline 739.2 & 5299.2 & 72.795604262895 & 884 & 688 & 90.38768103591 \\
\hline 412.8 & 1483.2 & 38.512335686115 & 456 & 372 & 47.81938070564 \\
\hline 441.6 & 3852.8 & 62.070927172067 & 536 & 384 & 77.071235600578 \\
\hline 433.6 & 1868.8 & 43.22961947554 & 480 & 392 & 53.676862155967 \\
\hline 355.2 & 6163.2 & 78.506050722221 & 480 & 288 & 97.478136817749 \\
\hline 590.4 & 19756.8 & 140.55888445772 & 744 & 360 & 174.52690644954 \\
\hline 595.2 & 28899.2 & 164.00975580739 & 720 & 312 & 203.64500912938 \\
\hline 441.6 & 10956.8 & 104.6747342963 & 592 & 368 & 129.97084909033 \\
\hline $\operatorname{sen}$ & $T=n$ & ne $7: 7 \mathrm{~nm}$ & ann & nen & $r 7 n$ \\
\hline
\end{tabular}

Figure 8: All results report set for throughput response

\begin{tabular}{l|lll|} 
Record \# & Throughput & $\begin{array}{l}\text { Lam1 } \\
\text { Utilization }\end{array}$ & $\begin{array}{l}\text { Lam2 } \\
\text { Utilization }\end{array}$ \\
\hline 1 & 380.0000 & 0.4975 & 0.7754 \\
2 & 520.0000 & 0.5890 & 0.6798 \\
3 & 460.0000 & 0.5976 & 0.8374 \\
\hline 4 & 420.0000 & 0.5719 & 0.8111 \\
5 & 600.0000 & 0.9397 & 0.9593 \\
\hline
\end{tabular}

Figure 9: Response values for an individual scenario

\subsection{Further analysis}

The Scenario Manager is designed to work with outside applications for open ended analysis. Currently Excel, JMP, and Minitab are directly supported. A text file export is also available that can be used with an even wider range of analysis programs (such as R).

The Export tab provides options for exporting the factors and responses to other software programs. Three different kinds of reports can be generated:

1. Complete results: Exports all of the factors and all of the response values for each run. There is one row or record of information for each simulation run. If there are multiple runs within a scenario, there will be multiple rows of results in the report for each scenario.

2. Displayed statistics: A report is generated that contains the currently selected report sets. There will be one row per scenario.

3. Complete statistics: A report is generated that contains all of the statistics (as if "All results" were selected for all of the responses) for all of the scenarios. Like the displayed statistics, there will be one row per scenario.

These reports can be sent to ExtendSim's internal database, Excel, JMP, Minitab or a text file. By using these tools more sophisticated analysis can be performed than is typically found in a simulation program alone. Figure 10 shows a sample analysis in Mintab. Here, we can clearly see the relationship between the model factors and model responses in a graphical format. This is just one of the many tools for scenario analysis that is available in a statistics package.

\section{CONCLUSION}

ExtendSim's Scenario Manager is a powerful tool for organizing and performing analysis on different model configurations. It encourages the modeler to explore the full range of model inputs and outputs. Because it is tightly integrated with other analysis programs, you can use any tool of choice to evaluate and present your simulation results. 


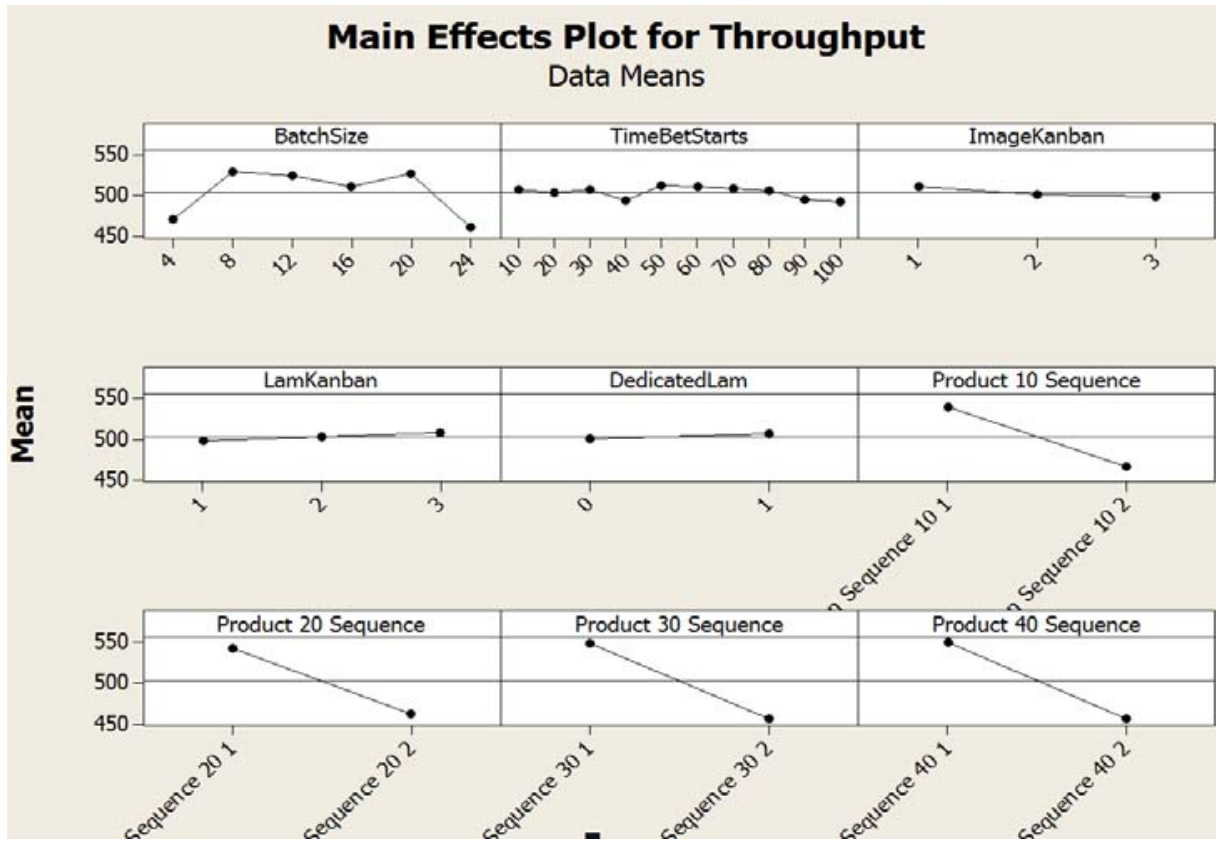

Figure 10: Model analysis in Minitab

\section{REFERENCES}

Diamond, B., D. Krahl, A. Nastasi, and P. Tag. 2010. "ExtendSim Advanced Technology: Integrated Database." In Proceedings of the 2010 Winter Simulation Conference, edited by B. Johansson, S. Jain, J. Montoya-Torres, J. Hugan, and E. Yücesan, 32-39. Piscataway, New Jersey: Institute of Electrical and Electronics Engineers, Inc.

Krahl, D., and J. S. Lamperti. 1997. “A Message-Based Discrete Event Simulation Architecture.” In Proceedings of the 1997 Winter Simulation Conference, edited by S. Andradottir, K. J. Healy, D. H. Withers, and B. L. Nelson, 1361-1367. Piscataway, New Jersey: Institute of Electrical and Electronics Engineers, Inc.

\section{AUTHOR BIOGRAPHY}

DAVID KRAHL is Vice President of Technical Sales with Imagine That, Inc. He received an MS in Project and Systems Management in 1996 from Golden Gate University and a BS in Industrial Engineering from the Rochester Institute of Technology in 1986. Mr. Krahl has worked extensively with a range of simulation programs including ExtendSim, SLAM II, TESS, Factor, AIM, GPSS, SIMAN, XCELL+ and MAP/1. A few of the companies that Mr. Krahl has worked with as a consultant and educator are Chrysler, Ford, Williams International, Tefen, Raytheon, and Boeing. He is actively involved in the simulation community. His email address is davek@extendsim.com and the Imagine That Inc. site is www.extendsim.com. 